\title{
Participación materna en la atención del niño hospitalizado
}

\author{
Francisco Barrera Q.1,2; Ana M. Sepúlveda B.1,2,3; Claudio Weber U.1; \\ Francisco Moraga M.1; Sonia Escobar M.4; Margarita Mejías J.4
}

\section{Mother's attendance to hospitalized children}

\begin{abstract}
Posent's company may contribute to improve qualily of hospilal core in the case of infants ond childien. Mothers of 100 patients aged 0 to 90 (mean 10.7 ) months who were admitted to the pediatric wards of a general usniversity associaled hospilal a metropolifan Sanfiogo, Chile, were asked to siay by their own choice with their children from 9 AM throughout 9 PM and were trained to help nurses in their ossistonce. Some end resulls of this experience were compored with lhose recorded from a similar number of children not allended by their mothers while in hospital. Significant decreases were observed in the frequency of toubles between patients and hospifal personnel -including prolessional staft-, nosocomial infecions $130 \mathrm{vs} 3 \%$. length of hospital stay was reduced by $20 \% 110$ to 8 days per bedl. Breasl leeding was preserved among all polients (38/38) cared for by their mothers in which it was previously present but in only 1 1.4\% 14/351 of controls. Nutrition improved in $91 \%$ of children of stay-in mothers vs $37 \%$ of control subjecls. Better underslanding of their children's problem was recorded in $95 \%$ of stay-in molhers. Mother's atlendonce lo hospitalized children seems to be odvarilogeous from both social and medical standpoints.
\end{abstract}

(Key wordst infant, child, hospitalization, hospilal-palient relations, malernal care, pediatric nursing.)

Con frecuencia se señala que en los hospitales se presta una atención de salud poco satisfactoria, a veces ineficiente y poco humanitaria, to que origina críticas no siempre bien fundamentadas, que dañan la relación médico-paciente y conducen a la obtención de información inadecuada del ambiente socioeconónico y familiar en el cual se desarrollan las enfermedades que causan hospitalización. A su vez, la separación del niño que sc hospitaliza y sus padres puede generar incertidumbre, angustia, hostilidad $y$ frustración.

La permancncia de la madre junto a su hijo enfermo durante la hospitalización puede ofrecer oportunidades únicas para educar a la familia en el manejo de los pacientes, disminuir los efectos

1. Médico, Servicio de Pediatria. Hospital Clínico San Borja-Arriarán.

2. Depunamento de Pediatría, Campos Centro. Facultad de Medicina, Universidad de Chile.

3. Residente becario de Pediatrín.

4. Enfermera universitaria, Servicio de Pediarría, Hospital Gíruco San Borja-Arriarán. sicológicos adversos provocados por la separación y mejorar la atención hospitalaria del los niflos enfermos.

EI estudio que se presenta a conlinuación se realizó en un servicio hospitalario de pediatria, con el proposito de evaluar los efectos de la participación de la madre en la atención del nifo hospitalizado. Los objetivos de dicho análisis fueron determinar si es factible la participación de la madre en la atención del niño hospitalizado y evaluar las posibles ventajas de dicha modalidad de atención hospitalaria en lo que respecta al estado de nutrición, tipo de alimentación recibi. da por los pacientes, educación y capacitación de las madres durante su contacto con el hospital y su personal.

\section{Material y Método}

Un equipo de médicos pediatras, enfermeras, nutricionistas, asistente social, asistente atdministrativo y técricos paramédicos consulto al mar -durante los meses de julio y octubre de 1991 - a 50 madres en la sala de espera del servicio de pediatria del Hospital Clínico San Borja-Arria- 
rán, demosirando que todas ellas deseaban pemanecer junto a su hijo hospitalizado, como resultado de lo cual se diseñó un prococolo de parícipación matema en la atención del niño hospitalizado.

El programa se inició en una unidad (lactantes B) de veinte camas - distribuida en cuatro salas-de las 120 dis ponibles del servicio de pediarra. Previamente se motiv6. informó y reorientó las funciones del equipo de salud en esta nueva modalidad de trabajo y, con el apoyo del Hospital Santa Ana -de Austrja-, se mejoró la habilitación de las salas de hospitalización, pintando las cunas en colores diferentes al blanco tradicional, vbicando sillas al lado de las cunas y disponiendo adecuada provisión de recolectores de material desechable, detergentes, jabón y toallas desechables. Además se dispuso de casilleros, servicio higiénico y un dclantal especialmentc diseñado para las madtcs. Parte del equipamiento requerido fue rescalado de la bodcga de excluidos. Las enfermeras prepararon un delantal para las madres y un manual de orienlación y educación para parti. cipar en el cuidado del niño enfermo. La dirección del hospital proporciono almuerzo y una industria de productos alimenticios donó colaciones de la tarde a las madtes participantes. El estudio se prolongó hasta que se obtuvieron registros de cien casos e igual número de controles.

Los pacientes objeto del estudio ingresaron desde el servicio de urgencia infantil a la unidad de ingreso del servicio de pediatría, permaneciendo en ella las primeras horas o días, para ser luego trasladados a la unidad de lactantes B. Como grupo control se eligieron niños de la misma edad con enfermedades similares y de severidad comparable, trasladados sinultánearnente con los anteriores desde la unidad de ingreso a otra (lactantes A) del mismo servicio, en la que no fueron acompañados de su madre durante la permanencia en el hospital. Los requisitos de ungreso al proyecto fucton niños de ambos sexos, desde recién nacidos hasta cuatro anos de edad, que cursaran una enfermedad leve a moderada, aguda o crónica, cuyas madres tuviesen interés en participar en el programa y se comprometiesen a permanecer $12 \mathrm{~h}$ diarias en el servicio, mientras durase la hospitalización. Dos madres fueron reliradas del proyecto, una de las cuales presentaba alteraciones psiquiatricas y otra por una incapacidad intelectual. Una madre ciega pudo participar en el proyecto, amamanlando a su hijo y colaboranda de acuerdo a sus pusibilidades.

Las madres legaban \& 1as 9:00 horas, recibiendo orientación sobre el programa. Luego enlraban a la sala donde estaba su hijo y se ponían a disposición de la enfermera del seclor y de la técnico paramédico de la sala, que les daban a conocer el manual de participación materna y reforzaban sus conocimientos en técnicas básicas, lavado de manos y alención del niño enfermo. Disponian de 45 minutos para almuerzo y 30 minutos para colación en la tarde, fuera det recinto clínico y se retiraban a las 21:00 h, excepto en casos de domicilio lejano que aconsejasen su retiro a las 20:00 $\mathrm{h}$. Excepcionalmente se acepio la presencia de personas que sustituyesen a la madre.

Al ingreso del paciente en la unidad, las madres fueron entrevistadas para identificar su percepción sobre la hospitalización, su grado de conocimientos de algunas técnicas y procedimientos básicos en la atención de su niño y las características del ambiente familiar. Al egreso realizó otra entrevista para precisar el impacto de la hospitalizacion en Ja familia, las conductas matemas, su comprensión del pro- blema de salud y una apreciación de la atención hospialaria. Con posterionidad al alta hospitalaria se hicieron visilas domiciliarias, para defínir el riesgo de reingreso, precisar la persistencia de las destrezas adquiridas y su proyección en la comnnidad, en lo que particípó la asistente social. Una nutricionista instruy f a la madre sobre alimentación durante la hospitalización (lactancia natural, otras alünentaciones) y sobre las indicaciones al alta.

\section{Resultados}

Los márgenes de edad de los niños participantes de ambos grupos fue desde el periodo de recién nacido hasta los 4 años, promedio de edad de 10,7 meses en el proyecto y 10,5 meses en el grupo control. Se observó claro predominio de nifios menores de un año, correspondicndo cllos a $62 \%$ en el proyecto y $66 \%$ en el gnupo control. En ambos grupos más de $70 \%$ (72 y $73 \%$ respectivamente) de los niños provenían del área central de salud.

El diagnostico de ingreso, en $54 \%$ del grupo en estudio y $51 \%$ de los controles, correspondió a infecciones de la via respiratoria baja, predominando el síndrome bronquial obstructivo asociado a bronconeumonia ( 28 y $23 \%$ respeclivamente) o de bronconeumonia (16 a $18 \%)$, el síndrome bronquial obstructivo puro se obscrvó en ambos grupos en proporciones de 10\%. El segundo diagnóstico en frecuencia fue síndrome diarreico en porcentaje de 20 y $25 \%$ respectivamente. En $26 \%$ de los casos en estudio y $24 \%$ del grupo control se registraron otros diagnósticos, propios de unidades de lactantes de alta complejidad, como fibrosis quística, enfermedad celíaca, atresia esofágica, síndrome de intestino corto, enfermedades metabólicas, afecciones neurológicas. En $32 \%$ de los niños atendidos con participación matema y $36 \%$ del grupo control se registraba alguna hospitalización previa.

En ambos grupos -estudio y control- el estado civil de casada era predominante en las madres (62\% y $59 \%$ ), seguido de la condición convivencia estable ( 15 y $20 \%$ ). Se observó un porcentaje similar e importante de madres solteras en ambas series (21 y $17 \%$ ). El mayor porcentaje de madres informó que su nivel de educación era básico 0 medio en ambas series ( 91 y 94\%). Si bien en los dos grupos $13 \%$ de las madres eran menores de 20 años, en $84 \%$ de los casos en estudio y $86 \%$ de controles las madres tenían entre 20 y 40 antos de edad. El número de hijos era uno a tres en 
$84 \%$ y $86 \%$ de las madres respectivamente y en ambos casos $97 \%$ de cllas tenían cuatro o menos hijos.

La duración de la hospitalización fue inferior a diez días en $75 \%$ de los niffos atendidos con la madre, en contraste con $56 \%$ del grupo control ( $\mathrm{p}<0,001)$, con promedios de duración de la estada hospitalaria de 8,1 y 12,9 dias respectivamente $(\mathrm{p}<0,001)$. Si se analiza sólo la permanencia de los niños en las respectivas unidades de lactante, sin considerar la breve estada de ingreso, las duraciones fueron 5,6 y 8,5 días.

La incidencia de infecciones intrahospitalarias entre los pacientes admitidos con su madre fue $3 \%$ y los controles $36 \%$, correspondiendo $33 \%$ de ellas a infecciones respiratorias agudas y $64 \%$ a diarrea aguda, siendo la prevalencia global de infección intrahospitalaria del servicio del orden de 10 a $20 \%$. En el grupo de niřos atendidos con participación materna un lactante mayor tenía evidencia de infecciones por Rotavirus, Escherichia colí y Shiguella simultáneamente, a pesar de lo cual no se registró un brote consecuente de infección intrahospitalaria en la sala. La disminución de la incidencia de infección intrahospitalaria entre los pacientes del estudio ocurrió de manera preponderante a expensas de las enfermedades de transmisión entérica.

El estado de la nutrición al ingreso era comparable en ambos grupos, aunque hubo mayor porcentaje de desnutrición leve en el grupo control y de desnutrición grave en la serie del estudio (tabla 1). Se observaron diferencias importantes en la evolución de la situación nutricional durante la hospitalización, pues $91 \%$ de los nirfos con madre participante aumentaron de peso y sólo

\section{Tabla 1}

Partícipación matema en la atención del nữî hospitalizado. Estado nutritivo al ingreso

\begin{tabular}{lrr}
\hline & $\begin{array}{c}\text { Proyecto } \\
\text { n (\%) }\end{array}$ & $\begin{array}{c}\text { Control } \\
\text { n (\%) }\end{array}$ \\
\hline $\begin{array}{l}\text { Estado notritivo } \\
\text { Eutrofia } \\
\quad \text { Sobrepeso } \\
\text { Desnutrición }\end{array}$ & 59 & 62 \\
Grado de desnutrición & 8 & 4 \\
D1 & 33 & 34 \\
D2 & & \\
D3 & 18 & 27 \\
\hline
\end{tabular}

6\% bajaron, en contraste con el grupo control en que el peso aumentó cn solo $37 \%$, mientras $60 \%$, por el contrario, mostraron tendencia a la baja ( $p<0,001$ ). Además en todos los niños del grupo con participación materna que al ingresar recibían alimentación natural (38/38) esta se mantuvo, en contraste con sólo $11,4 \%$ (4/35) en el grupo control y en ellos sólo en forma parcial.

Un miembro del equipo e fectuó una evaluación previa al ingreso de la madre y al momento del alta. Más de $90 \%$ de las madres que acompañaron a su hijo durante la hospitalización aprendieron o mejoraron sus conocimientos en cuidados básicos en la atención del niño enfermo, medidas de conforte general, higiene y alimentación. Se observó además la incorporación de técnicas y destrezas de mayor complejidad, tales como terapia de hidratación oral, alimentación por sonda nasogástrica o gastrostomía, uso de inhaladores y terapia de nebulización (tabla 2).

En una encuesta dirigida al equipo de salud, $86 \%$ de sus integrantes consideraron que la participación de la madre permitía acortar la estada hospitalaria y $92 \%$ que ella no interfería con el funcionamiento del servicio. Las madres estuvieron presentes en la visita clínica diaria del médico tratante, jefe de la unidad, interconsultas por equipo de especialistas, visita semanal del jefe de servicio, procedimientos de enfermería, kinesioterapia, colaborando eficazmente en muchas de cstas acciones. En algunas circunstancias justificadas en que se les solicito que abandonaran la sala, se logró su plena comprensión.

El seguimiento ambulatorio post-alta demostró un alto porcentaje de pacientes provenientes de otras áreas de la Región Metropolitana, superior a aquel informado al momento del ingreso al hospital.

\section{Comentario}

La participación de la madre en la atención del niño hospitalizado ha sido preconizada desde $1935^{1-4}$, sirn embargo sólo fue reconocida oficialmente como un derecho del niño hospitalizado en 1986 por la Comunidad Europea de Naciones. Ciertos aspectos de ella han sido parcialmente ratificadios en la Convención Internacional de los Derechos dcl Niño en 198916 .

En Europa, Estados Unidos y algunos países de Iberoamérica se han realizado experiencias 
Tabla 2

Participación materna en la atención đel niño hospitalizado. Evaluación del aprendizaje

\begin{tabular}{|c|c|c|c|c|}
\hline \multirow{2}{*}{ Destrezas y conocimientos } & \multicolumn{2}{|c|}{ Ingreso } & \multicolumn{2}{|c|}{ Egreso } \\
\hline & SI (\%) & No $(\%)$ & Si (\%) & No (\%) \\
\hline Tornar temperatura & 33 & 67 & 97 & 3 \\
\hline Medios para fiebre & 19 & 81 & 100 & - \\
\hline Alimentacion & 37 & 63 & 99 & 1 \\
\hline Higiene & 46 & 54 & 100 & - \\
\hline Cuidados básicos & 38 & 62 & 98 & 2 \\
\hline Prevencion enfermedades & 14 & 86 & 93 & $\overline{7}$ \\
\hline Nebulizar & 12 & 88 & 88 & 12 \\
\hline Uso inhalador & 28 & 72 & 92 & 8 \\
\hline Control signos vitales & 6 & 94 & 79 & 21 \\
\hline Alimentsción por SNG & 3 & 97 & 73 & 27 \\
\hline
\end{tabular}

permitiendo a la madre acompañar al hijo enfermo durante la hospitalización de éste, en algunas circunstancias, con objetivos de índole afectiva o práctica, como las atenciones básicas del niño, (alimentación, muda, baño, cambios de posición y vigilancia). En Chile, la visita de los padres a su hijo hospitalizado, está sujeta a normas que perturban la relación médico-paciente más allá de lo necesario. En la atención privada y en algunas instituciones universitarias, se pennite a la madre y a otros familiares acompañar al niño hospitalizado, preferentemente cuando se dispone de habitaciones individuales. Lo habitual en ellas es que la madre sólo cumpla un rol afectivo, de lactancia natural y colabore en la alimentación del niño. En algunas unidades (hematooncologia, tratamiento intensivo) de hospitales del sistema de salud y ciertos servicios de cirugía pediátrica se ha extendido el horario de visita, en el entendido que la mayor participación de los padres permitiría disminuir los días de estada, reducir el estrés perioperatorio, facilitar la cirugía ambulatoria y acortar la estada hospitalaria post intervencion.

Los temores iniciales sobre entorpecer el funcionamiento de la unidad clínica y aumento del riesgo de infección intrahospitalaria han sido disipados hace varios antos 7,8 .

Sin embargo, muchos padres no están en condiciones de integrarse a un servicio clínico que, además de su compleja labor asistencial, desarolle docencia de pre y postgrado e investigación. La angustia, la desesperación, la influencia de terceros y otros sentimientos que generan agre- sividad pueden entorpecer su participación y ocasionar dificultades menores. Una inadecuada supervisión médica y de enfermería sobre la madre participante puede a su vez permitir el desarrollo de conflictos de rol entre ésta y el personal técnico paramédico cuando ella detenta cientos rasgos de comportamiento desfavorables.

La incorporación de la madre a la atención de su niňo hospitalizado sobre una base programada permitiría aprovechar las ventajas descrítas en experiencias extranjeras, y deberian incidir en mayor humanización de la atención médica, disminución de los problemas en las salas de espera, mejoría en la calidad de la atención y enfoques mas integrales y positivos hacia la comunidad ${ }^{3,9-12}$. Es probable que en los diferentes hospitales y servicios clínicos ocurran diversas dificultades para desarrollar un programa con estas características, como hacinamiento de las salas, falta de comodidades mínimas para las madres, obstáculos culturales, geográficos, oposición de miembros del equipo de salud, incomprensión de niveles superiores de decisión. Sin embargo, nuestra opinión es que alrededor de $25 \%$ del total de niños hospitalizados puede permanecer al menos durante el dfa con su madre. Las dificultades mencionadas son solucionables. Los recursos necesarios son mínimos. El principal problema es nuestra propia resistencia al cambio. Puede interesarse a diversas instituciones dispuestas a colaborar y tal vez es una interesante oportunidad de acercar los servicios clínicos y la empresa privada. Se requerirá una nueva concepción arquitectónica de futuros servicios clínicos, permi- 
tiendo mayor acceso a la familia y lugares de esparcimiento para los nifos. La experiencia en nuestmo servicio, que a la fecha incluye ya a más de quinientas madres participantes, se ha realizado con dificultades mínimas. El ahorro de dias/ cama, que implica esta verdadera "escuela para madres de nif̂́os hospitalizados" y la mínima inversión requerida, son claras ventajas en la relación costo/beneficio. Si consideramos que el día/cama en nuestro servicio tiene un valor de $\$ 18000$ (US\$ 47), el programa descrito se ha traducido en un ahorro potencial de 500 días cama, equivalentes a $\$ 9000000$ (US $\$ 24000$ ) en menos de un año. En todo caso, conviene sefialar que en el Seminario de Derechos del Nino Hospitalizado, efectuado en septiembre de 1992 en Santiago de Chile, se ratificó como un derecho la permanencia de la madre junto a su hijo hospitalizado'12.

\section{Resumen}

La permanencia de la madre junto a su hijo hospitalizado permite, con minimas y solucionables dificultades, mejorar el funcionamiento de un servicio clínico. Se presenta un seguimiento prospectivo de 100 niños hospitalizados, que fueron acompañados voluntariamente por sus madres durante el dia, mientras permanecieron hospitalizados en el servicio de pediatría de un hospital general metropolitano, siguiendo un programa de participación materna en la atención de nifnos hospitalizados. Todas ellas fueron entrenadas de acuerdo a un manual de enfermería diseffado para tal proposito. La mayoria de las madres deseaban acompañar a su hijo hospitalizado. Los problemas habituales en sala de espera se redujeron de manera significativa. El promedio de estada hospitalaria disminuyo $20 \%$, de 10 a 8,1 días/cama. La frecuencia de infección intrahospitalaria se redujo de $36 \%$ en controles a sólo $3 \%$ en los pacientes estudiados. La lactancia natural se mantuvo en todos los casos que la recibían al ingreso y sólo en $11,4 \%$ de controles. El estado de nutrición al egreso mejoró en $91 \%$ de los niños cuidados por las madres y se deterioro en $60 \%$ de los controles. La tasa de reingreso se redujo y se obtuvieron modificaciones favorables de conducta y aprendizaje de nuevos conocimientos en el 95\% de las madres. Se detectaron algunas dificulcades menores, sobre todo en relación a la reestructuración de roles en el equipo de salud.

(Palabras clave: hospitalización de niños, participación materna.)

\section{Referencias}

1. McKay J: Consideraciones generales sobre la asistencia del niño enfermo. En Nelson W. Tratado de Pediatría, Editorial Interamericana, 1989: 176-179.

2. Alvarez H, Guerra M: La presencia de la madre en un Servicio de Pediatria. Labor psicosocial en el Hospital Migucl Pérez Carreño. Arch Dom Pedialr 1975; 11 : 1 12.

3. Barwin $H$ : The Hospital care of infants and children. J Pediatr 1951; 39: 383-390.

4. Barnard R: Place de la mère dans l'hospital d'enfants. La Revue de Pediatrie 1975; 11: 375-378.

5. Carta Europea de los niños hospilalizados. Parlamento Europeo. 14 abril 1986.

6. Convención Internacional de los Derechos del Niño. Asamblea general de las Naciones Unidas. Noviembre 1989.

7. Berhman $R$ : The new born infant. En Vaughn VC, McKay R, and Nelson W. Nelson textbook of pediatrics. Ed Saunders Company, Philadelphis, 1975; 329336.

8. Barrera F, Rebollo M: Asistencia nutricional en diarrea. Rev Chil Pediatr (supl) 1989; 60:35-38.

9. Suskind $R$ : E1 Servicio de apoyo nutricional: Un enfoque organizado de las unidades nutricionales del paciente pediátrico hospitalizado y ambulatorio. En Suskind R. Tratado de Nutrición en Pediatría Nueva York Ed. Raven Press books, 1985; 357-361.

10. Rosselot $J$ : Pediatria Infanul, Familia y Comunidad: Situación de la salud matemo infantil en America Latina y el Caribe. En Meneghello J. Pediatría Santiago de Chile, Ed. Medilerráneo, 1991: 30-48.

11. Grant J: Eslado Mundial de la Infancia 1992. Fondo de las Naciones Unidas para la Infancia (UNICEF). Ed. Adamson, Benson, Oxfordshire, Reino Unido.

12. Seminario: "Los Derechos del Niño Hospitalizado". Santiago de Chile 3-4-5 septiembre 1992. 